\title{
Informed Agents Integrating Data Mining and Agency
}

\author{
John Debenham and Simeon Simoff \\ University of Technology, \\ Sydney, Australia \\ \{debenham, simeon\}@it .uts.edu. au
}

\begin{abstract}
Summary. We propose that the key to building informed negotiating agents is to develop a form of agency that integrates naturally with data mining and information sources. These agent's take their historic observations as primitive, model their changing uncertainty in that information, and use that model as the foundation for the agent's reasoning. We describe an agent architecture, with an attendant theory, that is based on that model. In this approach, the utility of contracts, and the trust and reliability of a trading partner are intermediate concepts that an agent may estimate from its information model.
\end{abstract}

\section{Introduction}

The architecture of informed agents is designed to integrate naturally with information sources are their associated uncertainty. That is, we integrate:

- data mining - real-time data mining technology to tap information flows from the marketplace and the World Wide Web, and to deliver timely information at the right granularity.

- trading agents - intelligent agents that are designed to operate in tandem with the real-time information flows received from the data mining systems.

This paper describes an e-trading system that integrates these three technologies. The e-Market Framework is available on the World Wide Web ${ }^{1}$. This project aims to make informed automated trading a reality, and develops further the "Curious Negotiator" framework [1]. The data mining systems that have been developed for mining information both from the virtual institution and from general sources from the World Wide Web are described in Sec. 2. Intelligent agent that are built on an architecture designed specifically to handle real-time information flows are described in Sec. 3. Sec. 4 concludes.

\section{Data Mining}

We have designed information discovery and delivery agents that utilise text and network data mining for supporting real-time negotiation. This work has addressed the

\footnotetext{
${ }^{1}$ http://e-markets.org.au
}

Please use the following format when citing this chapter:

Debenham, J., Simoff, S., 2007, in IFIP International Federation for Information Processing, Volume 247, Artificial Intelligence and Innovations 2007: From Theory to Applications, eds. Boukis, C., Pnevmatikakis, L., Polymenakos, L., (Boston: Springer), pp. 165-173. 
central issues of extracting relevant information from different on-line repositories with different formats, with possible duplicative and erroneous data. That is, we have addressed the central issues in extracting information from the World Wide Web. Our mining agents understand the influence that extracted information has on the subject of negotiation and takes that in account.

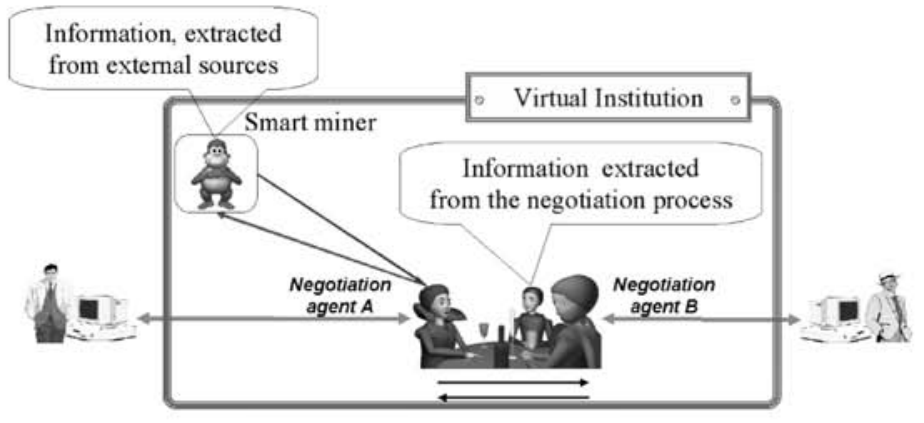

Fig. 1. The information that impacts trading negotiation

Real-time embedded data mining is an essential component of the proposed framework. In this framework the trading agents make their informed decisions, based on utilising two types of information (as illustrated in Figure 1): first, information extracted from the negotiation process (i.e. from the exchange of offers), and, second, information from external sources, extracted and provided in condensed form.

The embedded data mining system provides the information extracted from the external sources. The system complements and services the information-based architecture developed in [2] and [3]. The information request and the information delivery format is defined by the interaction ontology. As these agents operate with negotiation parameters with a discrete set of feasible values, the information request is formulated in terms of these values. As agents proceed with negotiation they have a topic of negotiation and a shared ontology that describes that topic. As the information-based architecture assumes that negotiation parameters are discrete, the information request can be formulated as a subset of the range of values for a negotiation parameter. The collection of parameter sets of the negotiation topic constitutes the input to the data mining system. Continuous numerical values are replaced by finite number of ranges of interest.

The data mining system initially constructs data sets that are "focused" on requested information, as illustrated in Figure 2. From the vast amount of information available in electronic form, we need to filter the information that is relevant to the information request. In our example, this will be the news, opinions, comments, white papers related to the five models of digital cameras. Technically, the automatic retrieval of the information pieces utilises the universal news bot architecture presented in [4]. Developed originally for news sites only, the approach is currently being extended to discussion boards and company white papers. 

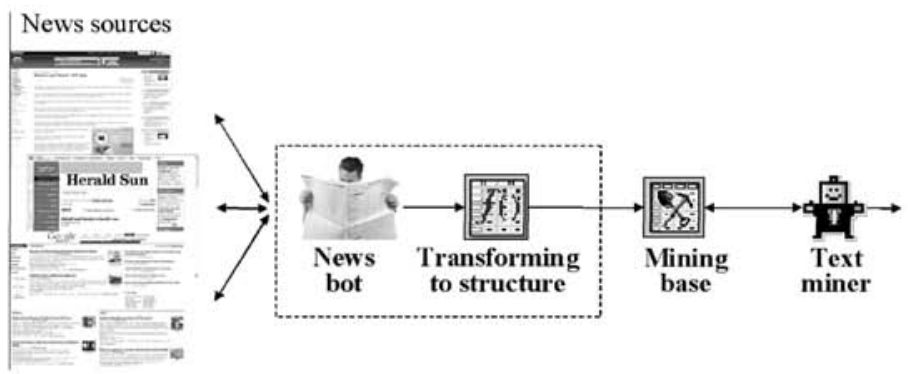

Fig. 2. The pipeline of constructing "focused" data sets

The "focused" data set is dynamically constructed in an iterative process. The data mining agent constructs the news data set according to the concepts in the query. Each concept is represented as a cluster of key terms (a term can include one or more words), defined by the proximity position of the frequent key terms. On each iteration the most frequent (terms) from the retrieved data set are extracted and considered to be related to the same concept. The extracted keywords are resubmitted to the search engine. The process of query submission, data retrieval and keyword extraction is repeated until the search results start to derail from the given topic.

The set of topics in the original request is used as a set of class labels. In our example we are interested in the evidence in support of each particular model camera model. A simple solution is for each model to introduce two labels - positive opinion and negative opinion, ending with ten labels. In the constructed focused data set, each news article is labelled with one of the values from this set of labels. An automated approach reported in [4] extends the tree-based approach proposed in [5].

Once the set is constructed, building the "advising model" is reduced to a classification data mining problem. As the model is communicated back to the informationbased agent architecture, the classifier output should include all the possible class labels with an attached probability estimates for each class. Hence, we use probabilistic classifiers (e.g. Naïve Bayes, Bayesian Network classifiers [6] without the min-max selection of the class output [e.g., in a classifier based on Naïve Bayes algorithm, we calculate the posterior probability $\mathbb{P}_{p}(i)$ of each class $c(i)$ with respect to combinations of key terms and then return the tuples $\left\langle c(i), \mathbb{P}_{p}(i)\right\rangle$ for all classes, not just the one with maximum $\mathbb{P}_{p}(i)$. In the case when we deal with range variables the data mining system returns the range within which is the estimated value. For example, the response to a request for an estimate of the rate of change between two currencies over specified period of time will be done in three steps: (i) the relative focused news data set will be updated for the specified period; (ii) the model that takes these news in account is updated, and; (iii) the output of the model is compared with requested ranges and the matching one is returned. The details of this part of the data mining system are presented in [7]. The currently used model is a modified linear model with an additional term that incorporates a news index Inews, which reflects the news effect on exchange rate. The current architecture of the data mining system in the e-market 
environment is shown in Figure 5. The $\left\{\theta_{1}, \ldots, \theta_{t}\right\}$ denote the output of the system to the information-based agent architecture.

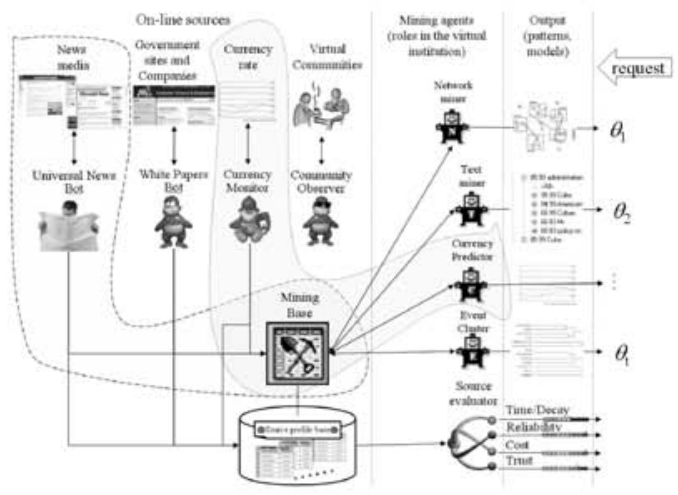

Fig. 3. The architecture of the agent-based data mining system

\section{Trading Agents}

We have designed a new agent architecture founded on information theory. These "information-based" agents operate in real-time in response to market information flows. We have addressed the central issues of trust in the execution of contracts, and the reliability of information [3]. Our agents understand the value of building business relationships as a foundation for reliable trade. An inherent difficulty in automated trading - including e-procurement - is that it is generally multi-issue. Even a simple trade, such as a quantity of steel, may involve: delivery date, settlement terms, as well as price and the quality of the steel. The "information-based" agent's reasoning is based on a first-order logic world model that manages multi-issue negotiation as easily as single-issue.

Most of the work on multi-issue negotiation has focussed on one-to-one bargaining - for example [8]. There has been rather less interest in one-to-many, multi-issue auctions - [9] analyzes some possibilities - despite the size of the e-procurement market which typically attempts to extend single-issue, reverse auctions to the multiissue case by post-auction haggling. There has been even less interest in many-tomany, multi-issue exchanges.

The generic architecture of our "information-based" agents is presented in Sec. 3.1. The agent's reasoning employs entropy-based inference and is described in [2]. The integrity of the agent's information is in a permanent state of decay, [3] describes the agent's machinery for managing this decay leading to a characterization of the "value" of information. Sec. 3.2 describes metrics that bring order and structure to the agent's information with the aim of supporting its management. 


\subsection{Information-Based Agent Architecture}

The essence of "information-based agency" is described as follows. An agent observes events in its environment including what other agents actually do. It chooses to represent some of those observations in its world model as beliefs. As time passes, an agent may not be prepared to accept such beliefs as being "true", and qualifies those representations with epistemic probabilities. Those qualified representations of prior observations are the agent's information. This information is primitive - it is the agent's representation of its beliefs about prior events in the environment and about the other agents prior actions. It is independent of what the agent is trying to achieve, or what the agent believes the other agents are trying to achieve. Given this information, an agent may then choose to adopt goals and strategies. Those strategies may be based on game theory, for example. To enable the agent's strategies to make good use of its information, tools from information theory are applied to summarize and process that information. Such an agent is called information-based.

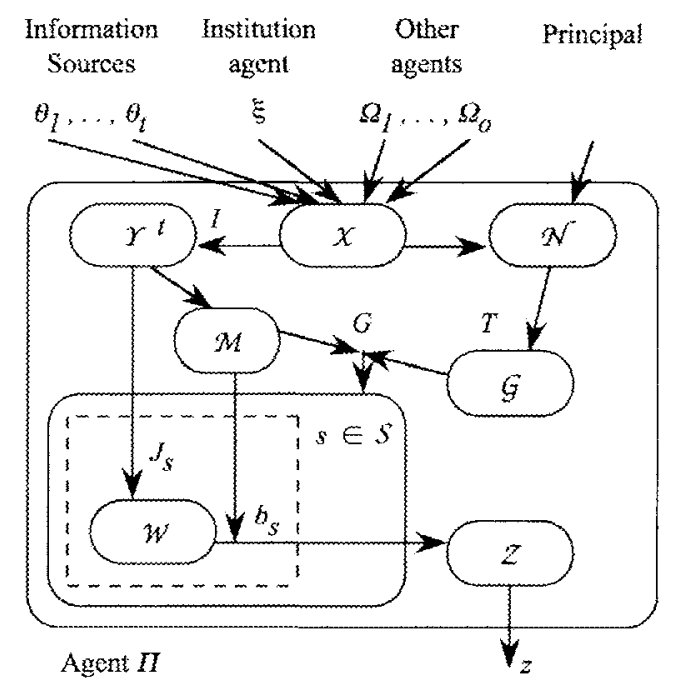

Fig. 4. Basic architecture of agent $\Pi$

An agent called $\Pi$ is the subject of this discussion. $\Pi$ engages in multi-issue negotiation with a set of other agents: $\left\{\Omega_{1}, \cdots, \Omega_{o}\right\}$. The foundation for $\Pi$ 's operation is the information that is generated both by and because of its negotiation exchanges. Any message from one agent to another reveals information about the sender. $I$ also acquires information from the environment - including general information sources - to support its actions. $\Pi$ uses ideas from information theory to process and summarize its information. $\Pi$ 's aim may not be "utility optimization" - it may not be aware of a utility function. If $\Pi$ does know its utility function and if it aims to opti- 
mize its utility then $\Pi$ may apply the principles of game theory to achieve its aim. The information-based approach does not to reject utility optimization - in general, the selection of a goal and strategy is secondary to the processing and summarizing of the information.

In addition to the information derived from its opponents, $\Pi$ has access to a set of information sources $\left\{\Theta_{1}, \cdots, \Theta_{t}\right\}$ that may include the marketplace in which trading takes place, and general information sources such as news-feeds accessed via the Internet. Together, $\Pi,\left\{\Omega_{1}, \cdots, \Omega_{o}\right\}$ and $\left\{\Theta_{1}, \cdots, \Theta_{t}\right\}$ make up a multiagent system. The integrity of $I$ 's information, including information extracted from the Internet, will decay in time. The way in which this decay occurs will depend on the type of information, and on the source from which it was drawn. Little appears to be known about how the integrity of real information, such as news-feeds, decays, although its validity can often be checked - "Is company X taking over company Y?" - by proactive action given a cooperative information source $\Theta_{j}$. So $I$ has to consider how and when to refresh its decaying information.

$\Pi$ has two languages: $\mathcal{C}$ and $\mathcal{L} . \mathcal{C}$ is an illocutionary-based language for communication. $\mathcal{L}$ is a first-order language for internal representation - precisely it is a first-order language with sentence probabilities optionally attached to each sentence representing $\Pi$ 's epistemic belief in the truth of that sentence. Fig. 4 shows a highlevel view of how $I I$ operates. Messages expressed in $\mathcal{C}$ from $\left\{\Theta_{i}\right\}$ and $\left\{\Omega_{i}\right\}$ are received, time-stamped, source-stamped and placed in an in-box $\mathcal{X}$. The messages in $\mathcal{X}$ are then translated using an import function $I$ into sentences expressed in $\mathcal{L}$ that have integrity decay functions (usually of time) attached to each sentence, they are stored in a repository $\mathcal{Y}^{t}$. And that is all that happens until $\Pi$ triggers a goal.

$\Pi$ triggers a goal, $g \in \mathcal{G}$, in two ways: first in response to a message received from an opponent $\left\{\Omega_{i}\right\}$ "I offer you $€ 1$ in exchange for an apple", and second in response to some need, $\nu \in \mathcal{N}$, "goodness, we've run out of coffee". In either case, $\Pi$ is motivated by a need - either a need to strike a deal with a particular feature (such as acquiring coffee) or a general need to trade. $\Pi$ 's goals could be short-term such as obtaining some information "what is the time?", medium-term such as striking a deal with one of its opponents, or, rather longer-term such as building a (business) relationship with one of its opponents. So $\Pi$ has a trigger mechanism $T$ where: $T:\{\mathcal{X} \cup \mathcal{N}\} \rightarrow G$.

For each goal that $I$ commits to, it has a mechanism, $G$, for selecting a strategy to achieve it where $G: \mathcal{G} \times \mathcal{M} \rightarrow \mathcal{S}$ where $\mathcal{S}$ is the strategy library. A strategy s maps an information base into an action, $s\left(\mathcal{Y}^{t}\right)=z \in \mathcal{Z}$. Given a goal, $g$, and the current state of the social model $m^{t}$, a strategy: $s=G\left(g, m^{t}\right)$. Each strategy, $s$, consists of a plan, $b_{s}$ and a world model (construction and revision) function, $J_{s}$, that constructs, and maintains the currency of, the strategy's world model $W_{s}^{t}$ that consists of a set of probability distributions. A plan derives the agent's next action, $z$, on the basis of the agent's world model for that strategy and the current state of the social model: $z=b_{s}\left(W_{s}^{t}, m^{t}\right)$, and $z=s\left(\mathcal{Y}^{t}\right) . J_{s}$ employs two forms of entropy-based inference:

- Maximum entropy inference, $J_{s}^{+}$, first constructs an information base $\mathcal{I}_{s}^{t}$ as a set of sentences expressed in $\mathcal{L}$ derived from $\mathcal{Y}^{t}$, and then from $\mathcal{I}_{s}^{t}$ constructs the world 
model, $W_{s}^{t}$, as a set of complete probability distributions using maximum entropy inference ${ }^{2}$.

- Given a prior world model, $W_{s}^{u}$, where $u<t$, minimum relative entropy inference, $J_{s}^{-}$, first constructs the incremental information base $\mathcal{I}_{s}^{(u, t)}$ of sentences derived from those in $\mathcal{Y}^{t}$ that were received between time $u$ and time $t$, and then from $W_{s}^{u}$ and $\mathcal{I}_{s}^{(u, t)}$ constructs a new world model, $W_{s}^{t}$ using minimum relative entropy inference.

\subsection{Valuing Information}

A chunk of information is valued first by the way that it enables $I$ to do something. So information is valued in relation to the strategies that $\Pi$ is executing. A strategy, $s$, is chosen for a particular goal $g$ in the context of a particular representation, or environment, $e$. One way in which a chunk of information assists $\Pi$ is by altering $s$ 's world model $W_{s}^{t}-$ see Fig. 4 . A model $W_{s}^{t}$ consists of a set of probability distributions: $W_{s}^{t}=\left\{D_{s, i}^{t}\right\}_{i=1}^{n}$. As a chunk of information could be "good" for one distribution and "bad" for another, we first value information by its effect on each distribution. For a model $W_{s}^{t}$, the value to $W_{s}^{t}$ of a message received at time $t$ is the resulting decrease in entropy in the distributions $\left\{D_{s, i}^{t}\right\}$. In general, suppose that a set of stamped messages $X=\left\{x_{i}\right\}$ is received in $\mathcal{X}$. The information in $X$ at time $t$ with respect to a particular distribution $D_{s, i}^{t} \in W_{s}^{t}$, strategy $s$, goal $g$ and environment $e$ is:

$$
\mathbb{I}\left(X \mid D_{s, i}^{t}, s, g, e\right) \triangleq \mathbb{H}\left(D_{s, i}^{t}\left(\mathcal{Y}^{t}\right)\right)-\mathbb{H}\left(D_{s, i}^{t}\left(\mathcal{Y}^{t} \cup I(X)\right)\right)
$$

for $i=1, \cdots, n$, where the argument of the $D_{s, i}^{t}(\cdot)$ is the state of $\Pi$ 's repository from which $D_{s, i}^{t}$ was derived. The environment $e$ could be determined by a need $\nu$ (if the evaluation is made in the context of a particular negotiation) or a relationship $\rho$ (in a broader context). It is reasonable to aggregate the information in $X$ over the distributions used by $s$. That is, the information in $X$ at time $t$ with respect to strategy $s$, goal $g$ and environment $e$ is:

$$
\mathbb{I}(X \mid s, g, e) \triangleq \sum_{i} \mathbb{I}\left(X \mid D_{s, i}^{t}, s, g, e\right)
$$

and to aggregate again over all strategies to obtain the value of the information in a statement. That is, the value of the information in $X$ with respect to goal $g$ and environment $e$ is:

\footnotetext{
${ }^{2}$ Given a probability distribution $\mathbf{q}$, the minimum relative entropy distribution $\mathbf{p}=$ $\left(p_{1}, \ldots, p_{I}\right)$ subject to a set of $J$ linear constraints $\mathbf{g}=\left\{g_{j}(\mathbf{p})=\mathbf{a}_{\mathbf{j}} \cdot \mathbf{p}-c_{j}=0\right\}, j=$ $1, \ldots, J$ (that must include the constraint $\sum_{i} p_{i}-1=0$ ) is: $\mathbf{p}=\arg \min _{\mathbf{r}} \sum_{j} r_{j} \log \frac{r_{j}}{q_{j}}$. This may be calculated by introducing Lagrange multipliers $\lambda: L(\mathbf{p}, \boldsymbol{\lambda})=\sum_{j} p_{j} \log \frac{p_{j}}{q_{j}}+$ $\boldsymbol{\lambda} \cdot \mathbf{g}$. Minimising $L,\left\{\frac{\partial L}{\partial \lambda_{j}}=g_{j}(\mathbf{p})=0\right\}, j=1, \ldots, J$ is the set of given constraints $\mathbf{g}$, and a solution to $\frac{\partial L}{\partial p_{i}}=0, i=1, \ldots, I$ leads eventually to $\mathbf{p}$. Entropy-based inference is a form of Bayesian inference that is convenient when the data is sparse [10] and encapsulates common-sense reasoning [11].
} 


$$
\mathbb{I}(X \mid g, e) \triangleq \sum_{s \in \mathcal{S}(g)} \mathbb{P}(s) \cdot \mathbb{I}(X \mid s, g, e)
$$

where $\mathbb{P}(s)$ is a distribution over the set of strategies for goal $g, \mathcal{S}(g)$, denoting the probability that strategy $s$ will be chosen for goal $g$ based on historic frequency data. and to aggregate again over all goals to obtain the (potential) information in a statement. That is, the potential information in $X$ with respect to environment $e$ is:

$$
\mathbb{I}(X \mid e) \triangleq \sum_{g \in \mathcal{G}} \mathbb{P}(g) \cdot \mathbb{I}(X \mid g, e)
$$

where $\mathbb{P}(g)$ is a distribution over $\mathcal{G}$ denoting the probability that strategy $g$ will be triggered based on historic frequency data.

\section{Conclusions}

A demonstrable prototype e-Market system permits both human and software agents to trade with each other on the World Wide Web. The main contributions described are: the broadly-based and "focussed" data mining systems, and the intelligent agent architecture founded on information theory. These technologies combine to give the foundation for our vision of the marketplaces of tomorrow.

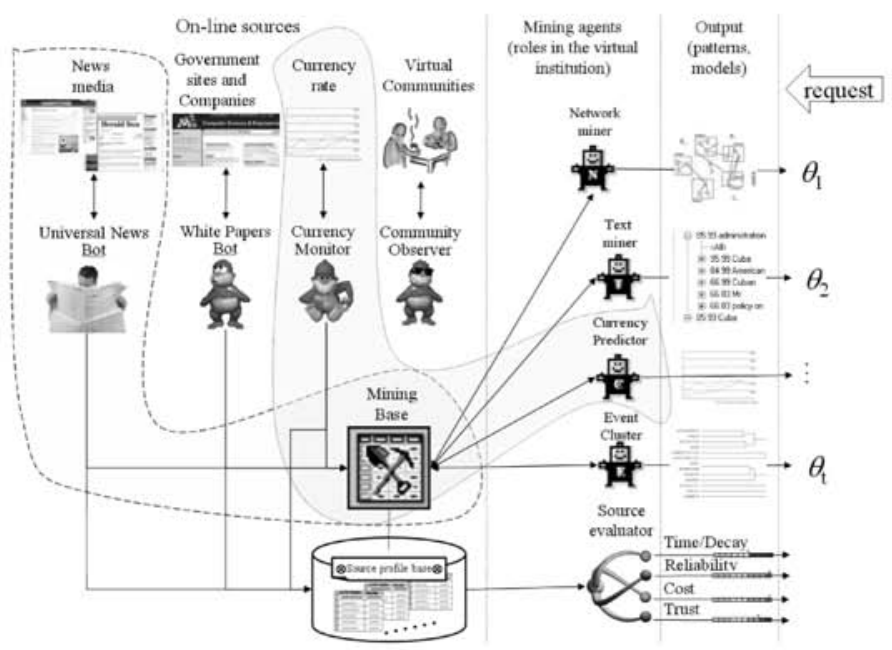

Fig. 5. The architecture of the agent-based data mining system

The implementation of these components is described in greater detail on our eMarkets Group Site ${ }^{1}$. The implementation of the data mining systems is notable for the way in which it is integrated with the trading agents - this enables the agents to 
dynamically assess the integrity of the various information sources. The implementation of the trading agents is greatly simplified by the assumption that preferences for each individual issue are common knowledge and are complementary for each a pair of traders [12]. This assumption, together with the use of coarse discrete representations of continuous variables, reduces the number of possible worlds and simplifies the minimum relative entropy calculations.

\section{References}

1. Debenham, J., Simoff, S.: An e-Market Framework for Informed Trading. In Carr, L., Roure, D.D., Iyengar, A., Goble, C., Dahlin, M., eds.: proceedings 15 th International World Wide Web Conference, WWW-2006, Edinburgh, Scotland (2006)

2. Debenham, J.: Bargaining with information. In Jennings, N., Sierra, C., Sonenberg, L., Tambe, M., eds.: Proceedings Third International Conference on Autonomous Agents and Multi Agent Systems AAMAS-2004, ACM Press, New York (2004) 664 - 671

3. Sierra, C., Debenham, J.: An information-based model for trust. In Dignum, F., Dignum, V., Koenig, S., Kraus, S., Singh, M., Wooldridge, M., eds.: Proceedings Fourth International Conference on Autonomous Agents and Multi Agent Systems AAMAS-2005, Utrecht, The Netherlands, ACM Press, New York (2005) 497 - 504

4. Zhang, D., Simoff, S.: Informing the Curious Negotiator: Automatic news extraction from the Internet. In: Proceedings 3rd Australasian Data Mining Conference, Cairns, Australia (2004) 55-72

5. Reis, D., Golgher, P.B., Silva, A., Laender, A.: Automatic web news extraction using tree edit distance. In: Proceedings of the 13th International Conference on the World Wide Web, New York (2004) 502-511

6. Ramoni, M., Sebastiani, P.: Bayesian methods. In: Intelligent Data Analysis. SpringerVerlag: Heidelberg, Germany (2003) 132-168

7. Zhang, D., Simoff, S., Debenham, J.: Exchange rate modelling using news articles and economic data. In: Proceedings of The 18th Australian Joint Conference on Artificial Intelligence, Sydney, Australia, Springer-Verlag: Heidelberg, Germany (2005)

8. Faratin, P., Sierra, C., Jennings, N.: Using similarity criteria to make issue trade-offs in automated negotiation. Journal of Artificial Intelligence 142 (2003) 205-237

9. Debenham, J.: Auctions and bidding with information. In Faratin, P., Rodriguez-Aguilar, J., eds:: Proceedings Agent-Mediated Electronic Commerce VI: AMEC. (2004) 15 - 28

10. Cheeseman, P., Stutz, J.: On The Relationship between Bayesian and Maximum Entropy Inference. In: Bayesian Inference and Maximum Entropy Methods in Science and Engineering. American Institute of Physics, Melville, NY, USA (2004) 445 - 461

11. Paris, J.: Common sense and maximum entropy. Synthese 117 (1999) 75 - 93

12. Sierra, C., Debenham, J.: Information-based agency. In: Proceedings of Twentieth International Joint Conference on Artificial Intelligence IJCAI-07, Hyderabad, India (2007) 1513-1518 